\title{
Low-Density Lipoprotein and Intracerebral Hematoma Expansion in Daily Alcohol Users
}

\author{
Gayle R. Pletsch $^{a} \quad$ Amelia K. Boehme ${ }^{c, d}$ Karen C. Albright ${ }^{c, e, f}$ \\ Christopher Burns $^{g} \quad$ T. Mark Beasley ${ }^{g}$ Sheryl Martin-Schild ${ }^{b}$
}

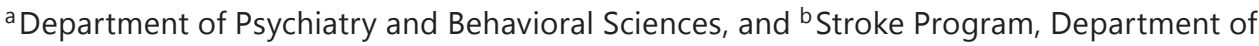
Neurology, Tulane University School of Medicine, New Orleans, La., and ' Department of Epidemiology, School of Public Health, dDepartment of Neurology, School of Medicine, e Health Services and Outcomes Research Center for Outcome and Effectiveness Research and Education (COERE), ${ }^{f}$ Center of Excellence in Comparative Effectiveness Research for Eliminating Disparities (CERED), Minority Health and Health Disparities Research Center (MHRC), and 9Department of Biostatistics, School of Public Health, University of Alabama at Birmingham, Birmingham, Ala., USA

Key Words

Low-density lipoprotein · Intracerebral hematoma · Hemorrhage $\cdot$ Expansion · Alcohol

\section{Abstract}

Background: Epidemiological studies suggest that the intracerebral hemorrhage (ICH) rate correlates with alcohol consumption. Alcohol leads to elevated blood pressure (BP) and inhibition of platelet aggregation. These factors could promote excessive bleeding. To our knowledge, in the setting of normal liver function tests, there are no studies that have systematically evaluated the relationship between daily alcohol use and hematoma expansion. The aim of this study is to compare the baseline ICH characteristics, frequency of hematoma expansion, and outcomes in patients with ICH who are daily alcohol users with those who are not daily alcohol users. Methods: A retrospective chart review was performed on consecutive patients who presented from July 2008 to July 2013 to the Tulane University Hospital in New Orleans, La., USA, with a spontaneous ICH. Ninety-nine patients who met these criteria were admitted. Patients who underwent hematoma evacuation were excluded. Hemorrhage volumes were calculated based on the $A B C / 2$ method. Low-density lipoprotein (LDL) was dichotomized into low $(<100 \mathrm{mg} / \mathrm{dl})$ and high $(\geq 100 \mathrm{mg} / \mathrm{dl})$ values. Comparisons were made using $\mathrm{t}$ tests, $\chi^{2}$ and nonparametric equivalents where appropriate. ICH growth in $24 \mathrm{~h}$ and LDL were evaluated using linear regression. Results: Of the 226 patients who met inclusion criteria, 20.4\% had a history of daily alcohol use. The average age was 61 years (range 19-94), 55.6\% of the patients were males, and $67.1 \%$ were of African American origin. Daily alcohol use was associated with 
Pletsch et al.: Low-Density Lipoprotein and Intracerebral Hematoma Expansion in Daily Alcohol Users

male gender, lower rate of home antihypertensive, higher presenting $\mathrm{BP}$, and lower platelet counts, but there was no difference in ICH characteristics, ICH growth, or clinical outcome. Daily alcohol use in patients with a low LDL level was associated with supratentorial location and trends for lower baseline Glasgow Coma Scale score, higher ICH score, and follow-up ICH volume, but no significant difference in significant hematoma expansion or clinical outcome except for a trend for higher mortality was found ( 25 vs. 9.5\%, $p=0.1311$ ) compared to patients with high LDL values. Conclusions: Our data suggest that neither chronic daily alcohol use nor a low LDL level in combination with daily alcohol use can be used to predict ICH growth. Daily alcohol use was associated with untreated hypertension and higher BP on presentation. A low LDL level in combination with daily alcohol use may be linked with larger and more severe ICH, but we were unable to demonstrate a relationship with hematoma expansion or poor clinical outcome. The role of LDL in vessel fragility and clot stabilization needs to be further explored before concluding that a low LDL level confers risk of bleeding.

(C) 2014 S. Karger AG, Basel

\section{Introduction}

Intracerebral hemorrhage (ICH) accounts for about $15 \%$ of stroke cases and is associated with a 30-day mortality rate exceeding 30\% [1]. Poor outcome in ICH patients has been linked to the neurologic deterioration seen with hematoma expansion [2]. Low low-density lipoprotein (LDL) and platelet inhibition, independent of hypertension, have been associated with hematoma expansion [3-6].

Consumption of excess alcohol has been linked to an increased risk of hemorrhagic stroke [7-9]. To our knowledge, in the setting of normal liver function tests, there are no studies that have systematically evaluated the relationship between daily alcohol use and hematoma expansion. In studies investigating the relationship between low LDL levels and ICH, a total cholesterol level of $<150 \mathrm{mg} / \mathrm{l}$ in the presence of active statin use was associated with greater hemorrhage volume and worse outcomes compared to statin users with higher cholesterol [10]. Another study found that LDL-cholesterol levels $<95 \mathrm{mg} / \mathrm{dl}$ were associated with increased hemorrhagic growth without significant difference in pre-ICH statin exposure in patients with and without hematoma growth [11].

Since a low LDL level and platelet inhibition have been independently shown to enhance hematoma expansion, we hypothesized that (1) patients who consumed alcohol daily would be more likely to experience hematoma expansion, and (2) patients with low LDL levels who consumed alcohol daily would be particularly prone to hematoma expansion.

\section{Methods}

A retrospective, observational study was performed on consecutive patients admitted to the Tulane Stroke Center, New Orleans, La., USA, with spontaneous ICH from July 2008 to July 2013. These patients were identified through our prospectively collected stroke registry. Patients were excluded if neurosurgical evacuation of the ICH was performed or the history of alcohol use was not clearly documented. They were not excluded for home use of anticoagulant or an elevated international normalized ratio on coagulation testing. Patients were divided into two groups: daily alcohol users and non-daily alcohol users. Daily alcohol use was defined as consumption of two or more alcohol equivalents on a daily basis, as reported by the patient and/or patient representative. The electronic medical record was reviewed to abstract current alcohol exposure. 
Pletsch et al.: Low-Density Lipoprotein and Intracerebral Hematoma Expansion in Daily Alcohol Users

Registry data included age, gender, race, initial blood pressure (BP), hypertensive history and medication compliance, Glasgow Coma Scale (GCS) score on initial evaluation, ICH score, National Institute of Health Stroke Scale (NIHSS) score as assessed by a NIHSS-certified healthcare provider, laboratory values, including blood counts, coagulation profile, and lipid panel, ICH volume and volume growth at $24 \mathrm{~h}$, discharge modified Rankin Scale (mRS) score, discharge disposition, and in-hospital mortality. All patients were admitted to the neurological intensive care unit. Lipids were measured after $12 \mathrm{~h}$ of fasting, but within $48 \mathrm{~h}$ of presentation.

ICH volume was calculated on baseline and follow-up computed tomography (CT) scans of the head using the $\mathrm{ABC} / 2$ method [12]. ICH score grading was performed at the time of admission by the admitting neurology resident and confirmed by a board certified vascular neurologist. Significant hematoma expansion was defined as an increase in volume by $\geq 33 \%$ [13] at $24 \mathrm{~h}$. Patients with hypertension, the primary etiology of spontaneous ICH, are at best with a moderate risk for cardiovascular and cerebrovascular events; therefore, we dichotomized the LDL level at $100 \mathrm{mg} / \mathrm{dl}$ based on the recommendation of the National Cholesterol Education Program Adult Treatment Panel. Functional outcome at the time of discharge was determined by a physician certified in the mRS score. Our primary end point was significant $(\geq 33 \%)$ hematoma expansion, and our secondary end point was any hematoma expansion.

Continuous variables without substantial deviations from a normal distribution were reported as means \pm standard deviation, and differences between the daily alcohol and nondaily alcohol users were analyzed with Satterthwaite's approximate t test. Continuous variables with non-normal distributions were reported as medians with ranges and analyzed with Wilcoxon's two-sample test for two-sided normal approximation. Categorical variables were analyzed using the $\chi^{2}$ test. The association between significant $(\geq 33 \%)$ hematoma expansion and daily alcohol use was modeled using logistic regression, while the association between hematoma expansion and daily alcohol use was modeled using linear regression. The regression models were repeated for the same outcomes using daily alcohol use in the setting of LDL $<100$ as the exposure. The relationship between ICH growth and LDL was modeled using a general linear model. As this was an exploratory analysis, no adjustments were made for multiple comparisons [14]. All tests were performed at a significance level of $\alpha=0.05$. This study was approved by our Institutional Review Board.

\section{Results}

Comparison of Patients with ICH in Daily Alcohol Users and Patients Who Do Not Consume Alcohol Daily

Of the 252 patients admitted with spontaneous ICH, 226 met criteria, of whom $20.4 \%$ had a history of daily alcohol use. The average age was 61 years (range 19-94), 55.6\% of the patients were males, and $67.1 \%$ were of African American origin. Those who were excluded for neurosurgical evacuation $(n=13)$ or undocumented alcohol use $(n=13)$ were not statistically different from those who were included with regard to GCS ( $p=0.1035)$ or baseline NIHSS ( $p=0.0883$ ), but they had higher initial volumes (34 vs. $10 \mathrm{ml}, \mathrm{p}=0.0195$ ). When compared to non-daily alcohol users, daily alcohol users were more likely to be of male gender (80.4 vs. $50.6 \%, \mathrm{p}=0.0003$ ), and to have a higher median BP upon admission (systolic BP of 190 vs. $173 \mathrm{~mm} \mathrm{Hg}, \mathrm{p}=0.0342$; diastolic BP of 115 vs. $110 \mathrm{~mm} \mathrm{Hg}, \mathrm{p}=0.0052$ ). Daily alcohol users were less likely to be taking BP medication at home (34.8 vs. $58.1 \%, \mathrm{p}=0.0138$ ) despite similar frequency of hypertension ( 69.6 vs. $85.0 \%, \mathrm{p}=0.0623$ ). As demonstrated in table 1 , there were no significant differences in race, mean LDL, mean triglycerides, use of anticoagulation or international normalized ratio, baseline GCS, or NIHSS in daily alcohol versus non- 
Pletsch et al.: Low-Density Lipoprotein and Intracerebral Hematoma Expansion in Daily Alcohol Users

Table 1. Baseline demographics and patient characteristics in the total patient sample

\begin{tabular}{lccc}
\hline Variable & $\begin{array}{c}\text { Daily alcohol } \\
\text { users }(\mathrm{n}=46)\end{array}$ & $\begin{array}{c}\text { Non-daily alcohol } \\
\text { users }(\mathrm{n}=180)\end{array}$ & p value \\
\hline Median age, years & $60(29-92)$ & $62(19-94)$ & 0.1116 \\
Male gender, $\mathrm{n}$ & $37(80.4)$ & $91(50.6)$ & 0.0003 \\
African American race, $\mathrm{n}$ & $29(67.4)$ & $118(66.7)$ & 0.3009 \\
History of hypertension, $\mathrm{n}$ & $32(69.6)$ & $146(85)$ & 0.0623 \\
Taking antihypertensive medication, $\mathrm{n}$ & $16(34.8)$ & $104(58.1)$ & 0.0138 \\
Median baseline SBP, mm Hg & $190(136-320)$ & $173(103-280)$ & 0.0342 \\
Median baseline DBP, mm Hg & $115(71-180)$ & $100(49-177)$ & 0.0052 \\
Median baseline GCS & $15(3-15)$ & $14(3-15)$ & 0.7437 \\
Median baseline NIHSS & $15(0-40)$ & $13(0-40)$ & 0.8887 \\
Median LDL, mg/dl & $97(19-217)$ & $101(34-245)$ & 0.4794 \\
Median triglycerides, mg/dl & $99(34-216)$ & $97(32-511)$ & 0.5426 \\
Median platelet count & $191(41-766)$ & $226(98-793)$ & 0.0051 \\
Warfarin use on admission, \% & $2(4.3)$ & $20(11.1)$ & 0.2630 \\
Median prothrombin time & $11.2(9.4-39.4)$ & $10.9(9.6-63.2)$ & 0.5653 \\
Median prothromboplastin time & $25.3(19.3-35.1)$ & $24.9(18.6-65.4)$ & 0.1509 \\
Median baseline ICH volume, ml & $9(0-72)$ & $9.1(0-186)$ & 0.8183 \\
Median ICH score & $1(0-4)$ & $1(0-6)$ & 0.4794 \\
Supratentorial location, $\mathrm{n}$ & $40(86.9)$ & $143(79.9)$ & 0.2725 \\
IVH, $\mathrm{n}$ & $20(43.5)$ & $77(42.8)$ & 0.9317 \\
Hydrocephalus, $\mathrm{n}$ & $10(21.7)$ & $52(28.9)$ & 0.3321 \\
Ventriculostomy, $\mathrm{n}$ & $8(17.4)$ & $20(11.1)$ & 0.2486 \\
\end{tabular}

Values in parentheses are percentages or ranges.

daily alcohol patients. Daily alcohol users had lower platelet counts than non-daily users (191 vs. $226, p=0.0051$ ). There were no significant differences in baseline or follow-up ICH volume, rate of intraventricular hemorrhage, external ventricular drain placement, hydrocephalus, supratentorial location or ICH scores (table 1). No significant differences in significant hematoma expansion, any hematoma expansion, or mean volume increase were found. Furthermore, there were also no significant differences in mean time to follow-up CT, discharge NIHSS, discharge mRS, discharge disposition, or in-hospital mortality, but lengths of stay was significantly prolonged in patients consuming daily alcohol ( $11 \mathrm{vs.} 7, \mathrm{p}=0.0156$ ) (table 2).

The crude model found a nonsignificant association between daily alcohol use and $>33 \%$ hematoma expansion $(\mathrm{OR}=1.26,95 \% \mathrm{CI} 0.55-2.86, \mathrm{p}=0.5858)$. Adjusting for gender, home $\mathrm{BP}$ medication use, and admission platelet count, the association remained, but was nonsignificant $(\mathrm{OR}=1.48,95 \%$ CI $0.36-6.17, \mathrm{p}=0.5874)$. The crude linear model did not find a significant association between hematoma expansion and daily alcohol use $(\beta=5.4, \mathrm{SE}=15.9$, $\mathrm{p}=0.7356$ ). After adjusting for differences between groups (gender, home BP medication use, admission platelet count), this remained nonsignificant $(\beta=3.3, \mathrm{SE}=7.8, \mathrm{p}=0.6742)$.

Comparison of Patients with ICH Who Use Alcohol Daily with Low versus High LDL Levels

LDL was not measured in 6 patients with chronic daily alcohol use and ICH, leaving 40 patients for comparison based on LDL dichotomized (20 patients with LDL $<100 \mathrm{mg} / \mathrm{dl}$ and 20 patients with LDL $\geq 100 \mathrm{mg} / \mathrm{dl}$ ). Daily alcohol users with a low LDL level $(<100 \mathrm{mg} / \mathrm{dl})$ were not significantly different from those with a high LDL level with respect to baseline demographics, history of hypertension, admission BP, prothrombin time, or platelet count. 
Pletsch et al.: Low-Density Lipoprotein and Intracerebral Hematoma Expansion in Daily Alcohol Users

Table 2. Patient outcomes of daily alcohol users versus non-daily alcohol users

\begin{tabular}{lccc}
\hline Outcomes & $\begin{array}{l}\text { Daily alcohol } \\
\text { users }(\mathrm{n}=46)\end{array}$ & $\begin{array}{l}\text { Non-daily alcohol } \\
\text { users (n=180) }\end{array}$ & p value \\
\hline Median follow-up ICH volume, ml & $10(1-167)$ & $10(0-402)$ & 0.8545 \\
Median time to follow-up CT, h & $7(3-28)$ & $7.8(4.5-30.5)$ & 0.5123 \\
Mean volume increase, ml & $1(-1.3$ to 138.5) & $0(-13$ to 346) & 0.1482 \\
Volume increase by $\geq 33, \mathrm{n}$ & $10(27)$ & $33(22.8)$ & 0.5854 \\
Median length of stay, days & $11(1-86)$ & $7(1-53)$ & 0.0156 \\
Median discharge NIHSS & $8(0-42)$ & $10(0-42)$ & 0.8228 \\
Median discharge mRS & $4(0-6)$ & $4(0-6)$ & 0.9193 \\
Death, n & $8(17.4)$ & $36(20)$ & 0.6901 \\
Disposition, n & $10(22.7)$ & $41(24.4)$ & 0.4856 \\
$\quad$ Home & $12(27.3)$ & $26(15.5)$ & $8(4.8)$ \\
$\quad$ Inpatient rehabilitation & $3(6.9)$ & $5(2.9)$ & \\
Skilled nursing/nursing home & $1(2.3)$ & $1(0.6)$ & \\
Long-term acute care & 0 & & \\
Hospice & & & \\
\hline
\end{tabular}

Values in parentheses are percentages or ranges.

Table 3. Characteristics of daily alcohol users with low versus high LDL

\begin{tabular}{lccc}
\hline Characteristics & $\begin{array}{l}\text { Daily alcohol users } \\
\text { with LDL }<100 \\
(\mathrm{n}=20)\end{array}$ & $\begin{array}{l}\text { Daily alcohol users } \\
\text { with LDL } \geq 100 \\
(\mathrm{n}=20)\end{array}$ & p value \\
\hline Median age, years & $61(29-92)$ & $52(39-79)$ & 0.2549 \\
Male gender, $\mathrm{n}$ & $19(95)$ & $15(75)$ & 0.5468 \\
African American race, $\mathrm{n}$ & $14(70)$ & $17(85)$ & 0.2464 \\
History of hypertension, $\mathrm{n}$ & $14(70)$ & $18(90)$ & 0.0515 \\
Taking antihypertensive medication, $\mathrm{n}$ & $10(50)$ & $7(35)$ & 0.5049 \\
Median baseline SBP, mm Hg & $180(136-320)$ & $218(140-248)$ & 0.2307 \\
Median baseline DBP, mm Hg & $103(74-180)$ & $123(85-170)$ & 0.1418 \\
Median baseline GCS & $13(3-15)$ & $15(4-15)$ & 0.1002 \\
Median baseline NIHSS & $16(0-38)$ & $13(0-33)$ & 0.4441 \\
Median triglycerides, mg/dl & $92(34-333)$ & $118(50-216)$ & 0.1331 \\
Median platelet count & $198(112-766)$ & $205(41-370)$ & 0.7415 \\
Median prothrombin time & $11.3(9.8-30.5)$ & $11.1(9.4-39.4)$ & 0.5691 \\
Median prothromboplastin time & $24.5(20.6-31.3)$ & $24.6(19.3-35.1)$ & 0.2235 \\
Supratentorial location, $\mathrm{n}$ & $20(100)$ & $16(80)$ & 0.0167 \\
Median baseline ICH volume, ml & $11(0-90.8)$ & $7(1-32)$ & 0.2479 \\
IVH, $\mathrm{n}$ & $10(50)$ & $8(40)$ & 0.2261 \\
Hydrocephalus, $\mathrm{n}$ & $6(30)$ & $5(25)$ & 0.2678 \\
Median ICH score & $2(0-4)$ & $1(0-3)$ & 0.1671 \\
Ventriculostomy, $\mathrm{n}$ & $5(25)$ & $5(25)$ & 0.2760 \\
\hline
\end{tabular}

Values in parentheses are ranges or percentages.

Daily alcohol users with a low LDL level had insignificantly larger median baseline ICH volumes ( 11 vs. $7 \mathrm{ml}, \mathrm{p}=0.2479$ ) and ICH scores ( 2 vs. $1, \mathrm{p}=0.1671$ ), but the frequencies of intraventricular hemorrhage and hydrocephalus were not significantly different in the two groups (table 3). As demonstrated in table 4, there was no difference in rate of significant $(\geq 33 \%)$ hematoma expansion at $24 \mathrm{~h}(25 \mathrm{vs} .21 \%, \mathrm{p}=0.3245)$ or any hematoma expansion 
Table 4. Patient outcomes of daily alcohol users with low versus high LDL

\begin{tabular}{lccc}
\hline Outcomes & $\begin{array}{c}\text { Daily alcohol users with } \\
\text { LDL }<100(\mathrm{n}=20)\end{array}$ & $\begin{array}{c}\text { Daily alcohol users } \\
\text { with LDL } \geq 100(\mathrm{n}=20)\end{array}$ & $\mathrm{p}$ value \\
\hline Median follow-up ICH volume, ml & $14.5(1.5-89.3)$ & $10(1-68)$ & 0.0676 \\
Median time to follow-up CT, $\mathrm{n}$ & $7(3.5-28)$ & $7(5-13)$ & 0.8463 \\
Mean volume increase, $\mathrm{h}$ & $1(-1.5$ to 21.9$)$ & $0.5(-1.3$ to 43$)$ & 0.2531 \\
Volume increase $>0$, $\mathrm{n}$ & $10 / 12(83.3)$ & $16 / 19(84.2)$ & 0.3764 \\
Volume increase by $\geq 33, \mathrm{n}$ & $3 / 12(25)$ & $4 / 19(21.1)$ & 0.3245 \\
Median length of stay, days & $9.5(1-86)$ & $11(3-40)$ & 0.2549 \\
Median discharge NIHSS & $11(0-42)$ & $10(0-42)$ & 0.8181 \\
Median discharge mRS & $5(0-6)$ & $4(0-6)$ & 0.2321 \\
Death, $\mathrm{n}$ & $6(30)$ & $2(10)$ & 0.1311 \\
Disposition, $\mathrm{n}$ & & $6 / 14(31.6)$ & 0.1877 \\
$\quad$ Home & $3 / 14(15.8)$ & $2 / 14(10.5)$ & $1 / 14(5.3)$ \\
$\quad$ Inpatient rehabilitation & $9 / 14(47.4)$ & $1 / 14(5.3)$ & \\
$\quad$ Skilled nursing/nursing home & $1 / 14(5.3)$ & $1 / 14(5.3)$ & \\
$\quad$ Long-term acute care & $1 / 14(5.3)$ & & \\
$\quad$ Hospice & 0 & & \\
\hline
\end{tabular}

Values in parentheses are percentages or ranges.

Fig. 1. Plot of ICH growth versus LDL level (mg/dl).

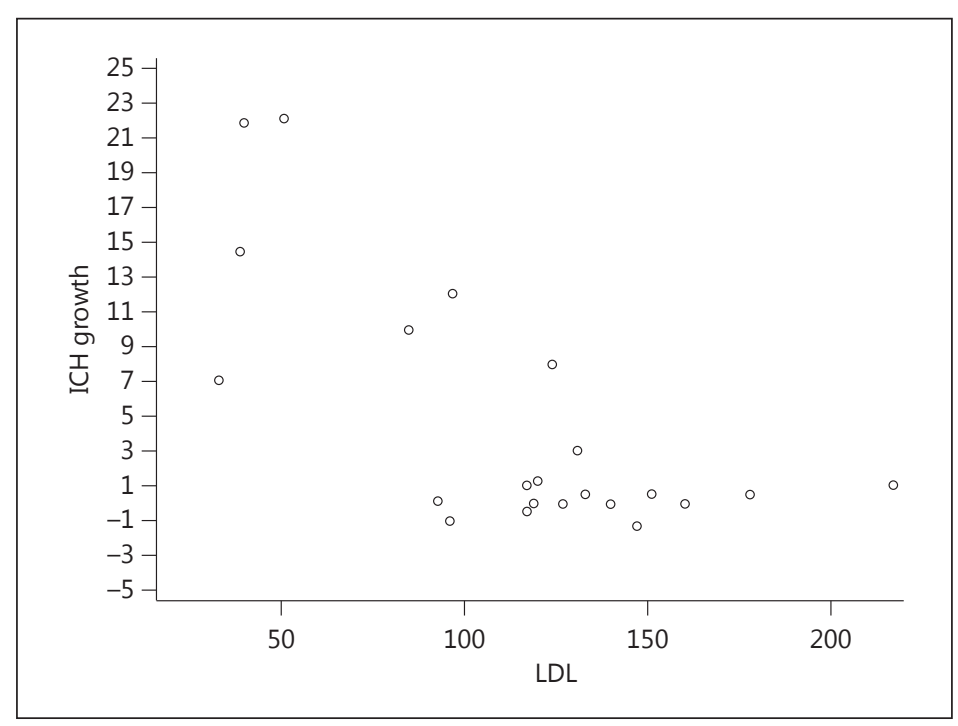

(83.3 vs. 84.2\%, p = 0.3764). Follow-up volumes ( $14.5 \mathrm{vs.} 10 \mathrm{ml}, \mathrm{p}=0.0676$ ) and mean volume increase ( 1 vs. $0.5 \mathrm{~h}, \mathrm{p}=0.2531$ ) were larger in patients with low LDL levels. No difference in discharge disposition or discharge NIHSS score was found. There were also no significant differences in mean time to follow-up CT, lengths of stay, discharge mRS and inpatient mortality rate.

The crude model found a nonsignificant association between hematoma expansion and low LDL levels in daily alcohol users $(\beta=1.7, \mathrm{SE}=13, \mathrm{p}=0.8966)$. After adjusting for initial ICH volume, this finding remained nonsignificant $(\beta=-1.5, \mathrm{SE}=14.3, \mathrm{p}=0.5730)$. Overall, ICH growth increased with progressively lower LDL measurements (fig. 1). 


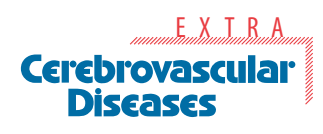

\begin{tabular}{l|l}
\hline Cerebrovasc Dis Extra 2014;4:1-8 \\
\hline DOI: $10.1159 / 000357611$ & $\begin{array}{l}\text { ( ) 2014 S. Karger AG, Basel } \\
\text { www.karger.com/cee }\end{array}$ \\
\hline
\end{tabular}

Pletsch et al.: Low-Density Lipoprotein and Intracerebral Hematoma Expansion in Daily Alcohol Users

\section{Discussion}

To the best of our knowledge, this is the first study to evaluate the relationship between daily alcohol intake, LDL and hematoma expansion. Previous studies have shown an association between heavy drinking and risk of ICH, but no current studies investigate hematoma expansion [15-17]. While we hypothesized that patients who consumed alcohol daily would be more likely to experience hematoma expansion, we did not find such an association between alcohol use and baseline or subsequent ICH volumes. We also failed to detect hematoma expansion amongst patients with low LDL levels who consumed alcohol daily compared to alcohol users who had high LDL levels.

Alcohol has been shown to lower the LDL level [18]. In our sample, the mean LDL level among dailyalcohol users was similar to patients who did not report daily alcohol consumption. We were unable to identify predictors of low LDL levels among alcohol users, possibly due to incomplete information on the actual grams of alcohol consumed on a daily basis and baseline nutritional status. Despite statistically significant difference in platelet count, the median platelet counts in the two groups were both within the normal range and not likely clinically meaningful. Furthermore, there was no difference in hematoma expansion, by any definition, comparing the chronic daily alcohol patients when the LDL level was not considered.

The impact of alcohol on BP elevation has been well described [6, 19-21]. In our sample, daily alcohol users had a higher systolic and diastolic BP upon admission. They reported taking their home BP medications less frequently than non-daily alcohol users. In addition, alcohol misuse may lead to medication noncompliance. In our sample, daily alcohol users presented with higher proportions of medication noncompliance and higher BP. Previous studies have shown that alcohol consumption reduces the protective effects of higher education [22].

Some epidemiological studies have reported that low cholesterol is associated with a higher incidence of ICH [23]. However, intensive lowering of cholesterol with high-dosed statins does not appear to increase the risk of ICH [24]. Prior studies investigating the risk of hemorrhagic transformation following an ischemic stroke have shown that a lower LDL level and total cholesterol are associated with an increased risk of hemorrhagic transformation [25]. We were unable to evaluate the potential association with statin use and hematoma expansion, since only three of our daily alcohol users were taking a statin prior to admission.

Our study had a number of limitations. It reflects a single center experience with a small number of patients, raising concern for generalizability. Our sample size may have impaired our ability to detect statistically significant differences between groups. We may have not been able to capture accurate social history on some of our sicker patients and others may have simply withheld information. We were unable to reliably quantify the alcohol use; therefore, we cannot determine a dose-response relationship with ICH growth, nor evaluate the relationship of binge drinking on ICH. Patients with daily alcohol use had higher baseline $\mathrm{BP}$; therefore, it is possible that difficulties in controlling the BP in the first $24 \mathrm{~h}$ confounded assessment of the hematoma expansion. Our admission order sets are standardized to include parameters and antihypertensive medications to maintain parameters, with physician call orders if parameters cannot be maintained. Use of the ABC/2 method, rather than semi-automated methods for measuring ICH volume, confers a degree of inaccuracy which may have impacted the validity of our findings [26]. Finally, we did not limit the study population to patients presenting in the first $6 \mathrm{~h}$ of symptom onset, when hematoma expansion is most likely to occur (last seen normal time unknown, $\mathrm{n}=49$; presentation within $6 \mathrm{~h}$ of symptom onset, $\mathrm{n}=121$ ). 


\section{Cerebrovascular Diseases}

\begin{tabular}{l|l}
\hline Cerebrovasc Dis Extra 2014;4:1-8 \\
\hline DOI: 10.1159/000357611 & $\begin{array}{l}\text { @ 2014 S. Karger AG, Basel } \\
\text { www.karger.com/cee }\end{array}$ \\
\hline
\end{tabular}

Pletsch et al.: Low-Density Lipoprotein and Intracerebral Hematoma Expansion in Daily Alcohol Users

\section{Conclusion}

Our study demonstrated no evidence to support that daily alcohol use with or without low LDL levels contributes to hematoma expansion after admission for spontaneous ICH. A larger prospective study, restricting the sample to patients presenting during the first hours when the risk for continued bleeding is high, is needed to further assess the relationships between alcohol use, LDL level, ICH volume and hematoma expansion.

\section{References}

1 Counsell C, Boonyakarnkul S, Dennis M, et al: Primary intracerebral hemorrhage in the Oxfordshire Community Stroke Project. Cerebrovasc Dis 1995;5:26-34.

2 Leira R, Dávalos A, Silva Y, et al: Early neurologic deterioration in intracerebral hemorrhage. Neurology 2004; 63:461-467.

3 Kase CS: Intracerebral hemorrhage: non-hypertensive causes. Stroke 1986;17:590-595.

-4 Sturgeon J, Folsom A, Longstreth WT Jr, Shahar E, Rosamond WD, Cushman M: Risk factors for intracerebral hemorrhage in a pooled prospective study. Stroke 2007;38:2718-2725.

5 Ramírez-Moreno JM, Casado-Naranjo I, Portilla JC, et al: Serum cholesterol LDL and 90-day mortality in patients with intracerebral hemorrhage. Stroke 2009;40:1917-1920.

6 Renaud SC, Ruf JC: Effects of alcohol on platelet functions. Clin Chim Acta 1996;246:77-89.

7 Ariesen MJ, Claus SP, Rinkel GJ, Algra A: Risk factors for intracerebral hemorrhage in the general population: a systematic review. Stroke 2003;34:2060-2065.

8 Mustacchi P: Risk factors in stroke. West J Med 1985;143:186-192.

-9 Beghi E, Boglium G, Cosso P, et al: Stroke and alcohol intake in a hospital population. A case-control study. Stroke 1995;26:1691-1696.

10 Miura K, Yoshii Y, Nakamura Y, Ikeda K: Clinicoradiological profile and serum lipid levels of intracerebral hemorrhage in prior statin users. Intern Med 2011;50:1385-1391.

-11 Rodriguez-Luna D, Rubiera M, Ribo M, et al: Serum low-density lipoprotein cholesterol level predicts hematoma growth and clinical outcome after acute intracerebral hemorrhage. Stroke 2011;42:2447-2452.

12 Kothari R, Brott T, Broderick J, et al: The ABCs of measuring intracerebral hemorrhage volumes. Stroke 1996; 27:1304-1305.

13 Brott T, Broderick J, Kothari R, et al: Early hemorrhage growth in patients with intracerebral hemorrhage. Stroke 1997;28:1-5.

14 Bender R, Lange S: Adjusting for multiple testing - when and how? J Clin Epidemiol 2001;54:343-349.

15 Hosomi N, Naya T, Ohkita H, Mukai M, Nakamura T, Ueno M, Dobashi H, Murao K, Masugata H, Miki T, et al: Predictors of intracerebral hemorrhage severity and its outcome in Japanese stroke patients. Cerebrovasc Dis 2009;27:67-74.

16 Peng GS, Yin SJ, Cheng CA, Chiu SW, Lee JT, Lin WW, Lin JC, Hsu YD: Increased risk of cerebral hemorrhage in Chinese male heavy drinkers with mild liver disorder. Cerebrovasc Dis 2007;23:309-314.

17 Herzig R, Urbanek K, Vlachova I, Krupka B, Gabrys M, Mares J, Schneiderka P: The role of chronic alcohol intake in patients with spontaneous intracranial hemorrhage: a carbohydrate-deficient transferrin study. Cerebrovasc Dis 2003;15:22-28.

18 Mukamal KJ, Mackey RH, Kuller LH, et al: Alcohol consumption and lipoprotein subclasses in older adults. J Clin Endocrinol Metab 2007;92:2559-2566.

19 Gillman MW, Cook NR, Evans DA, Rosner B, Hennekens CH: Relationship of alcohol intake with blood pressure in young adults. Hypertension 1995;25:1106-1110.

20 Numminen H, Syrjälä M, Benthin G, Kaste M, Hillbom M: The effect of acute ingestion of a large dose of alcohol on the hemostatic system and its circadian variation. Stroke 2000;31:1269-1273.

21 Alho H, Sillanaukee P, Kalela A, Jaakkola O, Laine S, Nikkari ST: Alcohol misuse increases serum antibodies to oxidized LDL and C-reactive protein. Alcohol 2004;39:312-315.

22 Grau AJ, Ling P, Palm F, Urbanek C, Becher H, Buggle F: Childhood and adult social conditions and risk of stroke. Cerebrovasc Dis 2012;33:385-391.

23 Noda H, Iso H, Irie F, et al: Low-density lipoprotein cholesterol concentrations and death due to intraparenchymal hemorrhage: The Ibaraki Prefectural Health Study. Circulation 2009;119:2136-2145.

24 Amarenco P, Bogousslavsky J, Callahan A III, et al: High-dose atorvastatin after stroke or transient ischemic attack. N Engl J Med 2006;355:549-559.

25 D'Amelio M, Terruso V, Famoso G, Ragonese P, Aridon P, Savettieri G: Cholesterol levels and risk of hemorrhagic transformation after acute ischemic stroke. Cerebrovasc Dis 2011;32:234-238.

26 Divani AA, Majida S, Luo X, et al: The ABCs of accurate volumetric measurement of cerebral hematoma. Stroke 2011;42:1569-1574. 